Original Research Paper

\title{
Multi-Level Image Thresholding via Nonlinear Fitting of the Histogram
}

\author{
Salah Ameer \\ Faculty of Applied Science and Technology, Humber College, Brampton, ON, Canada
}

\author{
Article history \\ Received: 24-09-2019 \\ Revised: 04-12-2019 \\ Accepted: 27-12-2019 \\ Email: salah.ameer@humber.ca
}

\begin{abstract}
This paper proposes a novel scheme for multi-level image thresholding. The 1D cumulative histogram is curve-fitted to a polynomial of nonlinear basis function, the hyperbolic tangent (tanh). The thresholds are the positions of the minima of the first (odd) derivative of the fitting polynomial, since the histogram is the derivative of the cumulative histogram. The tanh function is considered as an approximation to the integral of the Gaussian function. However, better results were obtained by fitting the derivative of the polynomial to the histogram. The scheme is a direct solution (solving a linear system) and does not require iterations or exhaustive search. Some results are presented to demonstrate the effectiveness of the proposed scheme.
\end{abstract}

Keywords: Image Thresholding, Histogram Fitting

\section{Introduction}

Multi-level thresholding of images is a significant step in image segmentation. Various schemes have been proposed in the literature, a good review can be found in Sezgin and Sankur (2004).

In the general image thresholding, the histogram is used as an approximation to the probability density function as employed by the two most common schemes, Otsu et al. (1979). And Kapur et al. (1985). In these cases and their extensions, the threshold is selected as a solution to an optimization problem for some objective function dependent on features extracted from the histogram. For the above mentioned schemes its variance and entropy respectively.

The aforementioned schemes can be generalized to multi-level thresholding as in Liu and Yu (2009). However, the computational price is too high. In addition, having many thresholds, the ensemble size for each region is reduced. This often results in inferior quality since statistics (or probability distribution, i.e. histogram) rely heavily on a large ensemble size.

To overcome the burden of exhaustive search, many researchers have proposed the use of metaheuristic schemes as in Oliva et al. (2018; Hu et al., 2019). These schemes, including the biologically inspired algorithms, may not be the best solutions as demonstrated by Balabanian et al. (2017; Merzban and Elbayoumi, 2019; Olugbara et al., 2015).

As a remedy to incorporate local information into the histogram, Patra et al. (2014). suggested an energy function to be found at each gray level exploring similarity (or dissimilarity) using a $3 \times 3$ window centered at each pixel.

There are many measures see (Sezgin and Sankur (2004). to evaluate the performance of a thresholding scheme. However, in some applications, a subjective decision may be preferred.

The Gaussian function is typically used to approximate the statistics of regions in the image histogram. However, due to nonlinearity, direct fitting has been avoided. To overcome this shortcoming, this paper proposes approximating the erf function (the integral of the Gaussian function) instead. The tanh or arctan functions can be used as approximations to the erf function.

This research proposes few formulations to nonlinearly fit the 1D (cumulative) histogram. Since a polynomial of tanh function basis can be used to fit the cumulative histogram, the derivative of this polynomial is a good candidate to fit the histogram. Other nonlinear functions such as arctan and erf (error function) can also be used. However, the computational cost is higher. The thresholds are then found as the positions of the minima of first (odd) derivatives of the nonlinear polynomial.

\section{Proposed Algorithm}

Without loss of generality, the gray levels $\mathrm{g}$ are normalized to the interval $[0,1]$.

To better condition the data, the following modification is used:

$$
G=\frac{g-c}{\sigma}
$$


where, $c$ is the image mean and $\sigma$ is the standard deviation of the image. Let's construct a polynomial to fit the cumulative histogram having the form:

$$
\begin{aligned}
& Y=\sum_{i=1}^{N} a_{i} x^{1} \\
& x=\tanh (G)
\end{aligned}
$$

Now we are ready to fit the histogram $h$ by minimizing the following error function:

$$
\begin{aligned}
& s=\sum_{k=1}^{L}\left\{y_{k}^{\prime}-h_{k}\right\}^{2} \\
& y_{k}^{\prime}=\left.\frac{\partial y}{\partial G}\right|_{G=G_{k}}
\end{aligned}
$$

$L$ is the number of gray levels in the histogram. It should not be forgotten that the derivative of $x$ is (1$x^{2}$ ). The variables to be found are the polynomial coefficients $a_{i}$, turning the problem into a linear system of order $N$.

Obviously, the higher the value of $N$ the better the approximation. $N$ should be large enough to capture the variations in the histogram. Unfortunately, increasing the value of $N$ can result in an ill-conditioned linear solution due to high magnitudes of the coefficients.

A small regularization term can be added to better condition the linear system. Hence, our objective function to optimize is:

$$
s=\sum_{k=1}^{L}\left\{\sum_{i=1}^{N} i a_{i}\left(1-x^{2}\right) x^{i-1}-h_{k}\right\}^{2}+\delta \sum_{i=1}^{N} a_{i}^{2}
$$

$\delta$ is a small number ( $\sim 0.0005$ can be used).

Values of $\mathrm{N}>20$ tend to produce similar outcomes, however, a more thorough investigation is needed to find the optimum value of $N$ and $\delta$. It should be emphasized that the value of $N$ is vaguely related to the number of thresholds since the scheme generates an automatic value for the number of regions.

To avoid biasing towards larger regions, the logarithm of the histogram is used, resulting in the following optimization:

$$
\begin{aligned}
& s=\sum_{k=1}^{L}\left\{\sum_{i=1}^{N} i a_{i}\left(1-x^{2}\right) x^{l-1}-H_{k}\right\}^{2}+\delta \sum_{i=1}^{N} a_{i}^{2} \\
& H_{k}=\log \left(1+h_{k}\right)
\end{aligned}
$$

In addition, the histogram or its log version should be normalized such that its sum is equal to one. This step is necessary since the tanh function is bounded by one. An illustrative procedure is shown in Table 1.

Other versions can be designed, keeping in mind the necessary modification required for the derivative in Equation (5), using:

$$
\begin{aligned}
& x=\tan ^{-1}(G) \\
& x=\operatorname{erf}(G)
\end{aligned}
$$

Histogram minima are good candidates for threshold values (Sezgin and Sankur, 2004). Hence, the minima of $y^{\prime}$ are the threshold candidates in this work. However, higher order odd derivatives (e.g. $y^{(3)}$ or $y^{(5)}$ ) may be used to further explore small regions having their distributions merged or falling under the distribution of a bigger region. A precaution should be maintained to the fact that higher order derivatives will tend to be more descriptive of the basis function (tanh, $\tan ^{-1}$ and erf) than the actual data.

In fact, regular polynomial fitting can also be used with acceptable performance. However, low values of $N$ should be used for a better conditioned linear system.

In some cases, better performance can be obtained by not including gray levels having zero valued histogram. This can help in reducing the computation time for sparse histograms. However, it has marginal influence on the performance.

Two evaluation schemes are considered in this paper to compare with recent results in the field. The first is the SSIM given by, Wang et al. (2004):

$\operatorname{SSIM}=\frac{\left(2 \mu_{x} \mu_{y}+C_{1}\right)}{\left(\mu_{x}^{2}+\mu_{y}^{2}+C_{1}\right)} \frac{\left(2 \sigma x y+C_{2}\right)}{\left(\sigma_{x}^{2}+\sigma_{y}^{2}+C_{2}\right)}$

where, $x$ and $y$ stand for original and segmented images, $\mu$ is the mean, $\sigma^{2}$ is the variance and $\sigma_{x y}$ is the covariance between $x$ and $y$.

The second evaluation scheme is the traditional RMSE given by:

$R M S E=\sqrt{\frac{1}{\|x\|} \sum_{m, n}\left(x_{m n-} y_{m n}\right)^{2}}$

where, $\|x\|$ is the cardinality of the set.

\section{Experimental Results}

Extensive testing have been performed on about 80 images. From a statistical point of view, no clear evidence to favor one of the functions ( $\tanh , \tan ^{-1}$ and erf) over the others.

The proposed scheme is a global thresholding scheme and hence it lacks the benefit of incorporating local information. In addition, the number of thresholds is automatically generated.

To illustrate the proposed algorithm, the standard image pepper is used as shown in Fig. 1. Figure 2-5 show segmentation results for $\tanh , \tan ^{-1}$, erf and regular 
polynomial with $\mathrm{N}=5,10,15,20,25$ using the minima of the first derivative of the fitting polynomial.

As can be seen from Fig. 2-5, the higher the order the more the capturing of the description of regions in the image, in other words, better approximation to the minima of the histogram. However, the number of regions may increase.

The $\log$ of the histograms for $\mathrm{N}=5$ and 25 for the tanh function case are shown in Fig. 6. Other functions exhibit similar performance.

As expected the regular polynomial is not preferred for high orders (>16 experimentally) due to illconditioned linear system.

Higher order derivatives can sometimes (erf is an exception) produce better results, with the cost of more regions. Figure 7-9 show segmentation results for tanh, $\tan ^{-1}$ and erf with $\mathrm{N}=5,10,15,20,25$ using the minima of the third derivative of the fitting polynomial.

Obviously, the computations using the tanh function is less involved since the derivative is sech $^{2}$ which is $1-\tanh ^{2}$. For this reason and for space saving purposes, future comparisons will be performed using the tanh function.

In terms of computational complexity, histogram generation requires $\mathrm{O}(\mathrm{S})$ where $\mathrm{S}$ is the image size. The generation of the linear system requires $\mathrm{O}(\mathrm{NL})$ plus $\mathrm{O}\left(\mathrm{N}^{2} \mathrm{~L}\right)$ that can be performed off-line for the system matrix. Solving the linear system typically is $\mathrm{O}\left(\mathrm{N}^{2}\right)$. At last, finding the minima is of $\mathrm{O}(\mathrm{L})$ complexity.
To save the effort in reproducing results of other schemes, the results of the proposed scheme where compared to that of Olugbara et al. (2015). where an extensive comparison is made with some meta-heuristic schemes following the between class variance optimization originally proposed by Otsu (1979). Olugbara et al. (2015). claim the improved performance of their scheme. Comparing that to the proposed scheme inherently implies comparison with all the references used in Olugbara et al. (2015).

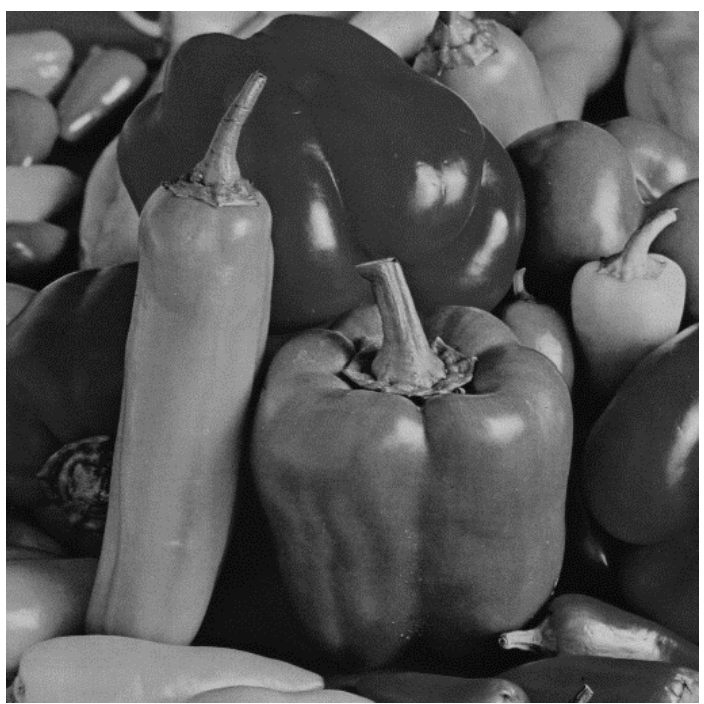

Fig. 1: Test image pepper
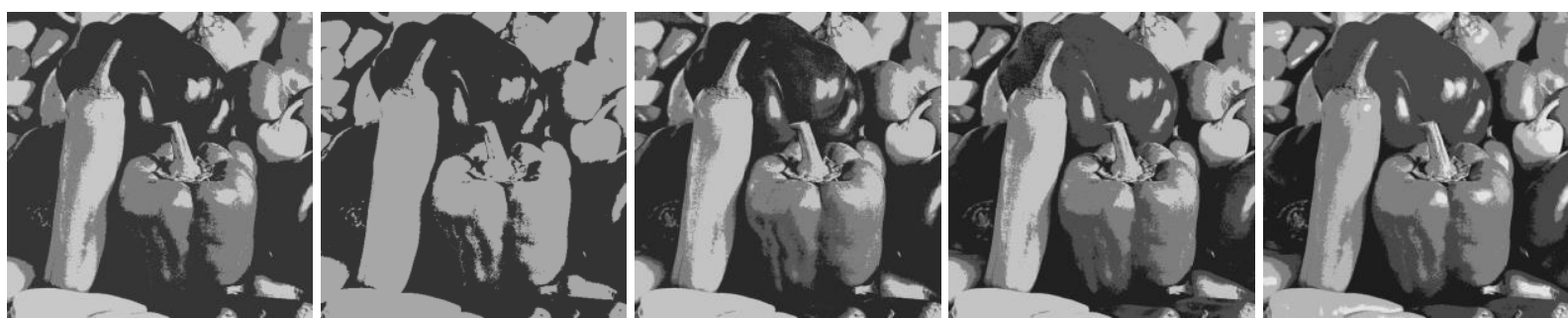

Fig. 2: Segmentation using the first derivative applying tanh function with $\mathrm{N}=5,10,15,20,25$. (left to right) having \#regions/SS IM of: $3 / 0.934,2 / 0.864,4 / 0.948,4 / 0.958$ and $5 / 0.972$ respectively
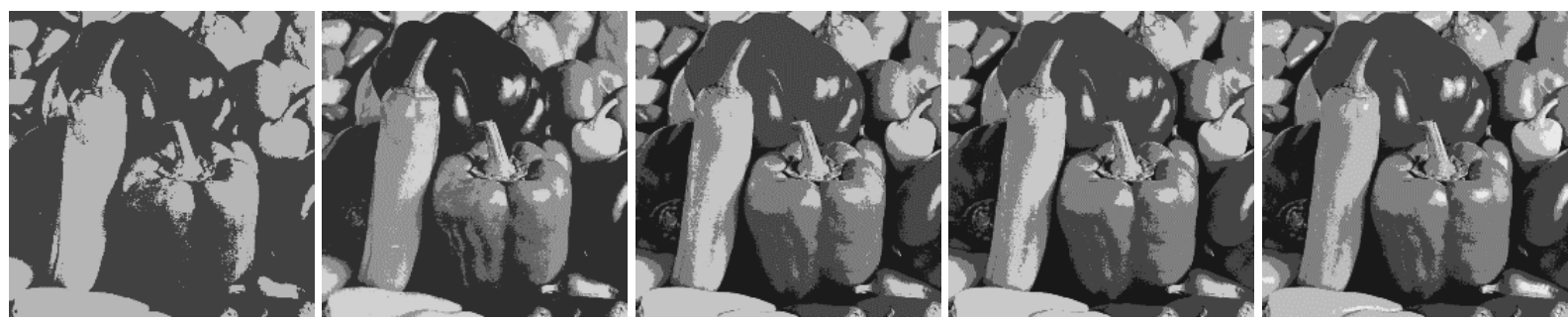

Fig. 3: Segmentation using the first derivative applying $\tan ^{-1}$ function with $N=5,10,15,20,25$. (left to right) having \#regions/SSIM of: $2 / 0.865,4 / 0.953,4 / 0.960,4 / 0.960$ and $5 / 0.968$ respectively 

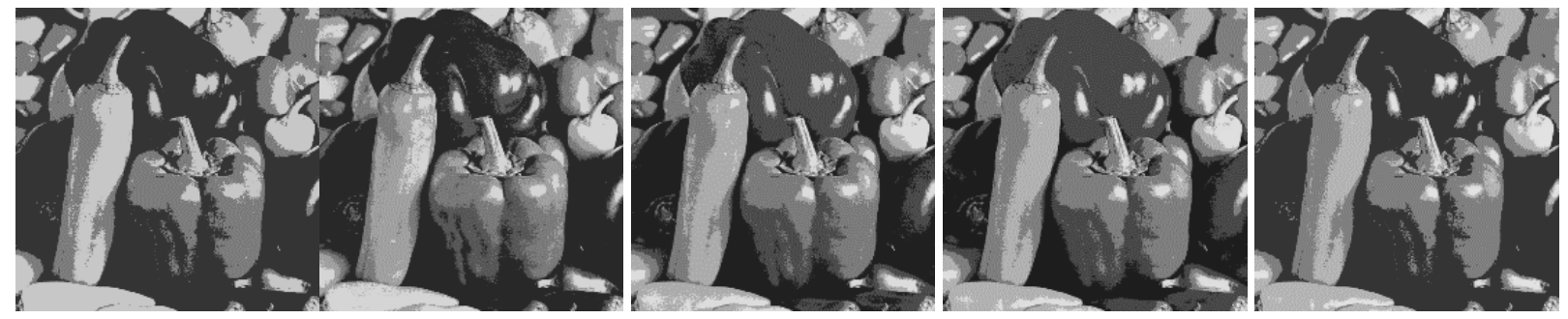

Fig. 4: Segmentation using the first derivative applying erf function with $\mathrm{N}=5,10,15,20,25$. (left to right) having \#regions/SSIM of: $3 / 0.934,5 / 0.965,5 / 0.973,5 / 0.973$ and $4 / 0.945$ respectively
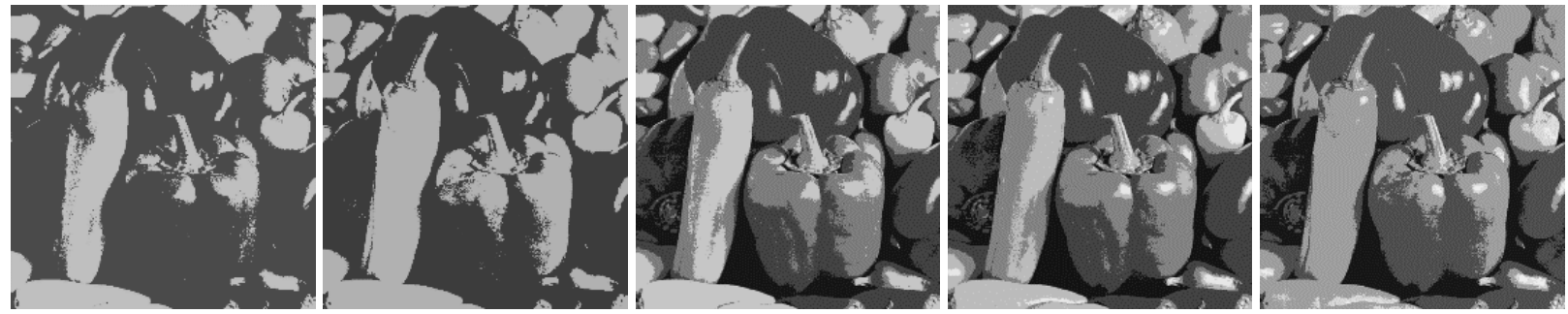

Fig. 5: Segmentation using the first derivative applying regular polynomial with $\mathrm{N}=5,10,15,20,25$. (left to right) having \#regions/SSIM of: 2/0.824, 2/0.872, 5/0.958, 6/0.966 and 7/0.937 respectively
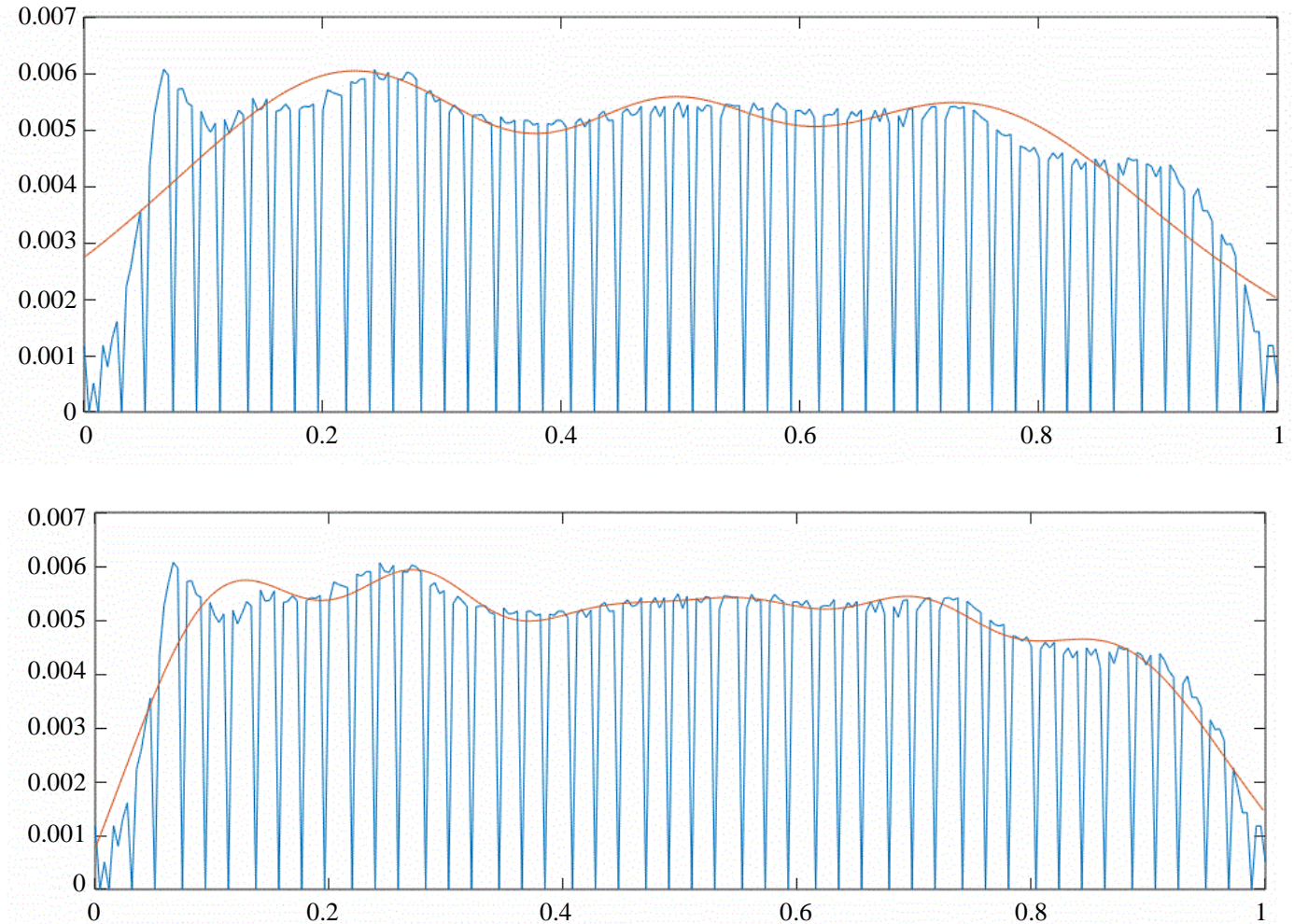

Fig. 6: Original histogram (blue) and fitted histogram (red) applying a polynomial of order $\mathrm{N}$ with tanh as the basis function. $\mathrm{N}=$ 5 (top) and $\mathrm{N}=25$ (buttom)

Figure 10-14. illustrate the proposed segmentation on some images from Berkley Segmentation Database (BSD) Martin et al. (2001). Polynomial having tanh as the basis function are used. Figure 10-14. show the number of regions, linear system order(N), derivative order and value of SSIM plus the value of SSIM 
obtained by Olugbara et al. (2015). The images are converted to gray scale in the same manner performed by the reference.

Figure 10-14 clearly indicate the high quality of the proposed scheme. Although it is slightly inferior in SSIM to that of Olugbara et al. (2015). nevertheless it can produce similar results with a smaller number of regions. It should be emphasized that Olugbara et al. (2015). reported higher performance compared to some other meta heuristic schemes, putting the performance of proposed scheme in higher rank compared to these schemes.
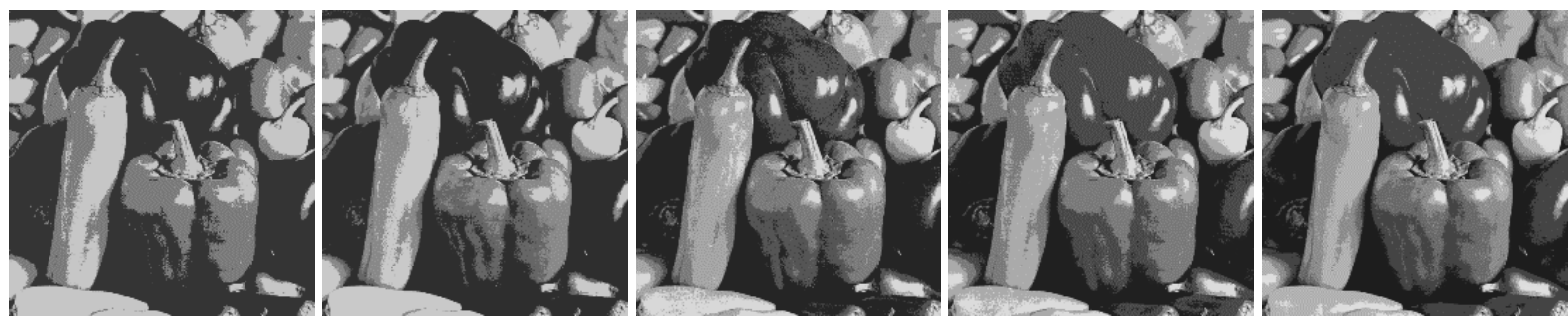

Fig. 7: Segmentation using the third derivative applying tanh function with $N=5,10,15,20,25$. (left to right) having \#regions/SSIM of: 3/0.934, 4/0.950, 5/0.969, 5/0.973 and 6/0.983 respectively
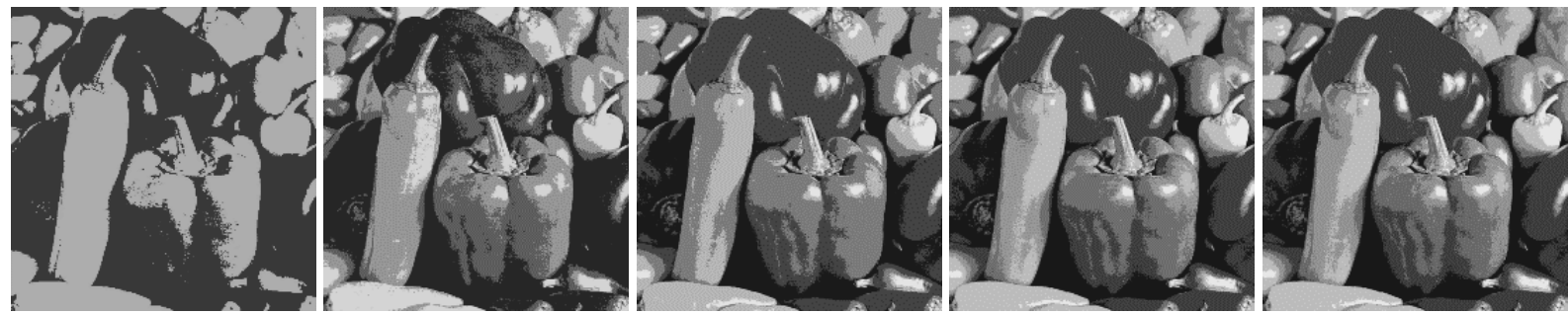

Fig. 8: Segmentation using the third derivative applying $\tan ^{-1}$ function with $\mathrm{N}=5,10,15,20,25$. (left to right) having \#regions/SSIM of: $2 / 0.872,4 / 0.955,5 / 0.971,6 / 0.981$ and 6/0.982 respectively
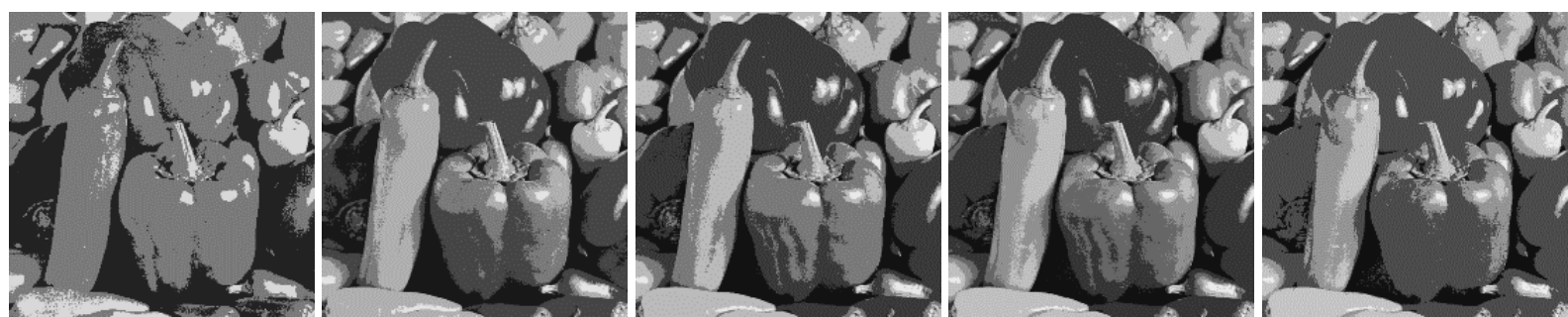

Fig. 9: Segmentation using the third derivative applying erf function with $\mathrm{N}=5,10,15,20,25$. (left to right) having \#regions/SSIM of: $3 / 0.825,5 / 0.969,5 / 0.963,6 / 0.976$ and $5 / 0.926$ respectively
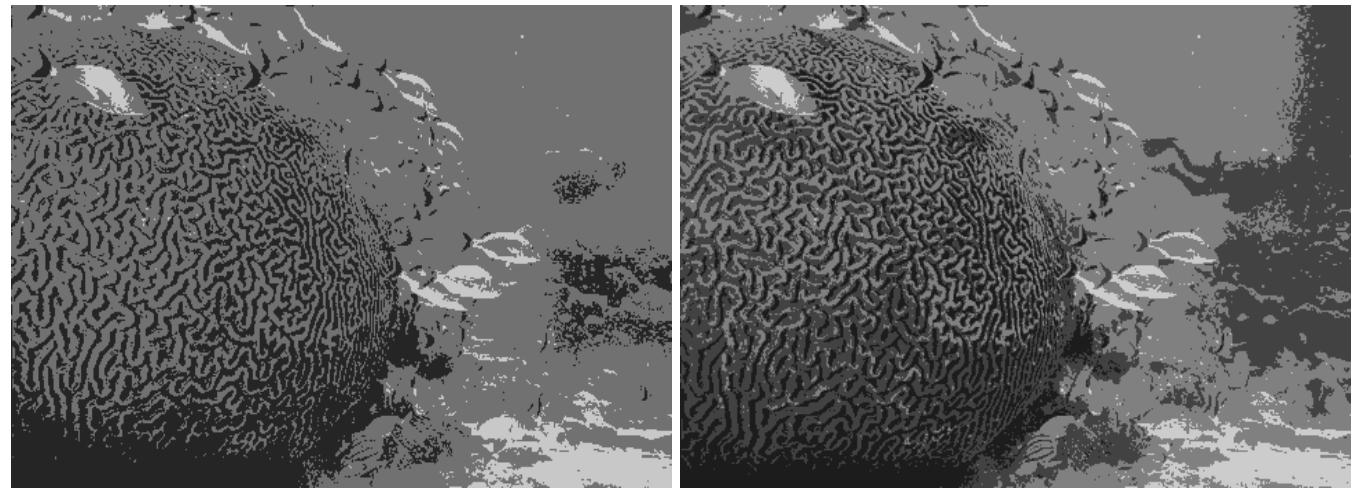

Fig. 10: Segmentation results applying tanh function for image 45077. (left to right) having \#regions/SSIM/N/derivative of: $3 / 0.825 / 14 / 1$ and 4/0.901/30/1. Ref $=3 / 0.898$ 
Salah Ameer / American Journal of Applied Sciences 2019, 16 (12): 336.345 DOI: 10.3844/ajassp.2019.336.345
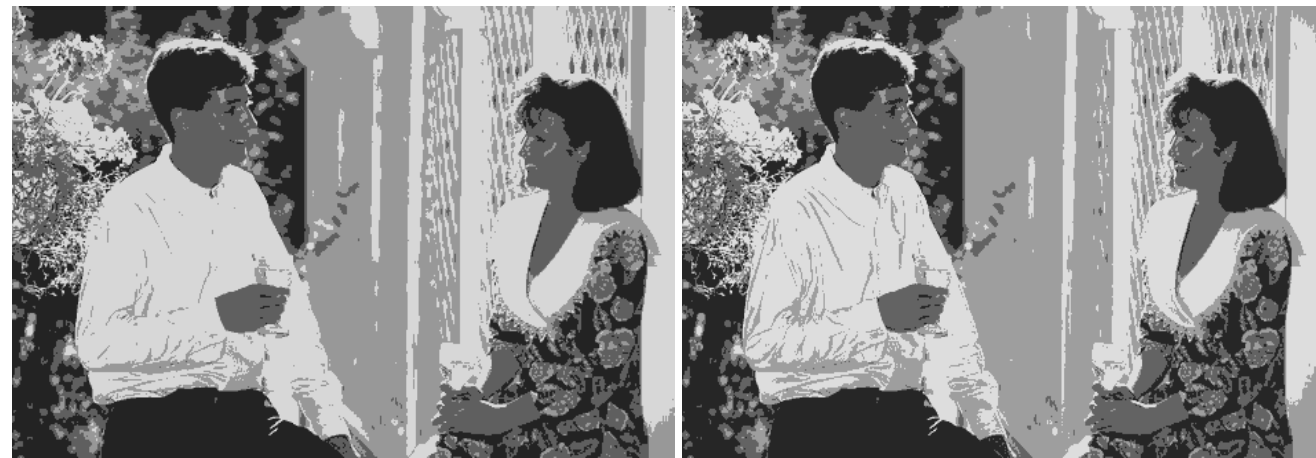

Fig. 11: Segmentation results applying tanh function for image 157055. (left to right) having \#regions/SSIM/N/derivative of: $4 / 0.967 / 19 / 1$ and $4 / 0.968 / 9 / 3$. Ref $=4 / 0.942$
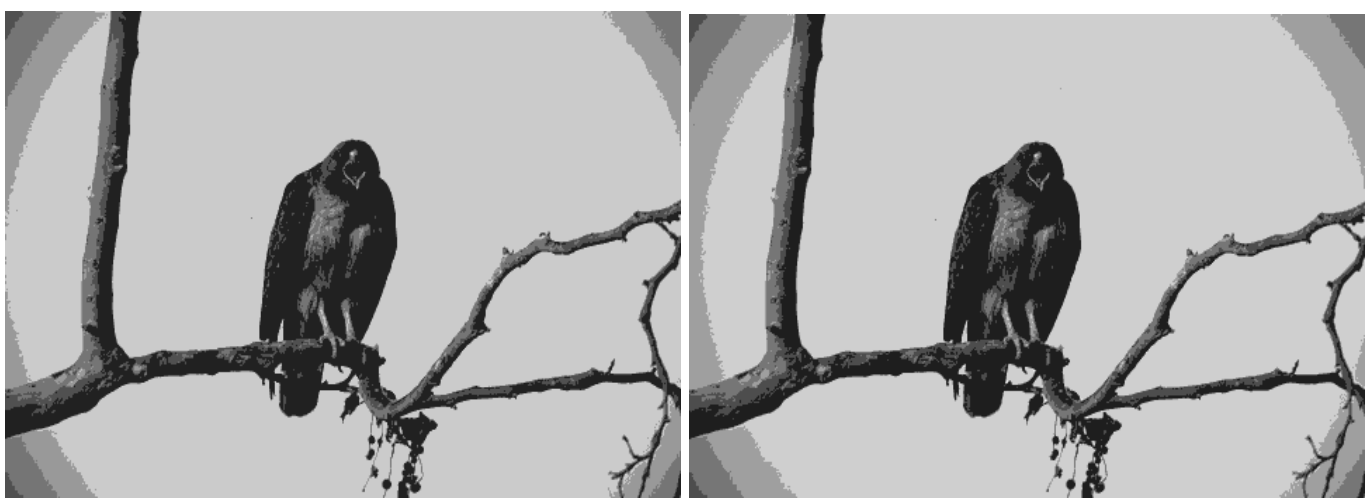

Fig. 12: Segmentation results applying tanh function for image 42049. (left to right) having \#regions/SSIM/N/derivative of: $5 / 0.983 / 20 / 1$ and 6/0.987/20/3. Ref $=6 / 0.989$
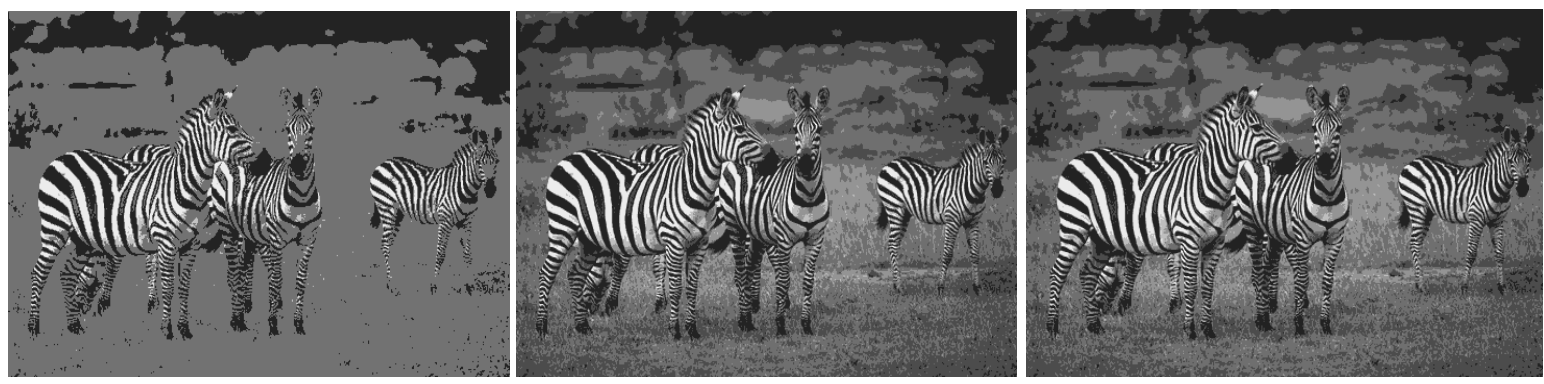

Fig. 13: Segmentation results applying tanh function for image 253027. (left to right) having \#regions/SSIM/N/derivative of: $4 / 0.877 / 20 / 1,6 / 0.973 / 20 / 3$ and $7 / 0.973 / 20 / 5$. Ref $=7 / 0.979$
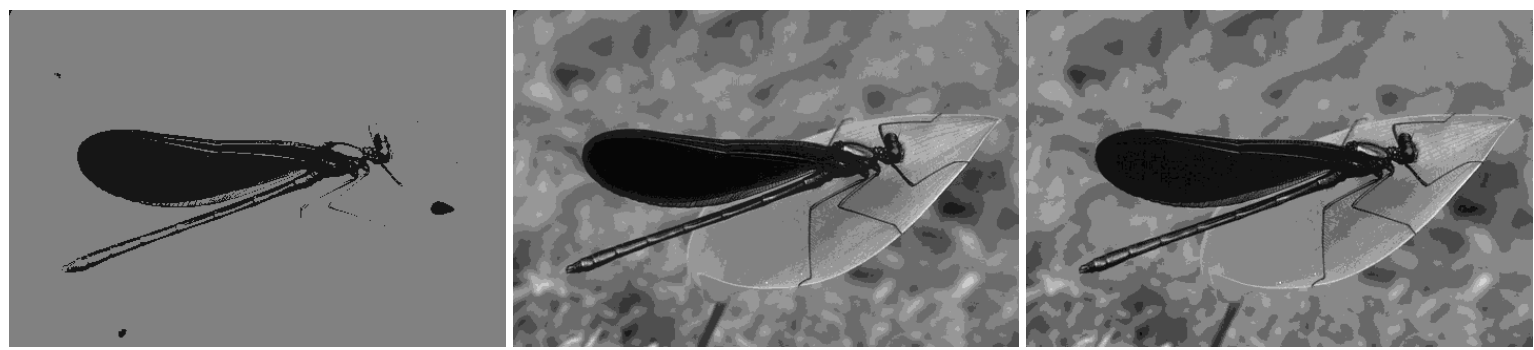

Fig. 14: Segmentation results applying tanh function for image 35070. (left to right) having \#regions/SSIM/N/derivative of: 2/0.800/20/1, 11/0.986/30/11 and 13/0.976/40/11. Ref = 13/0.994 


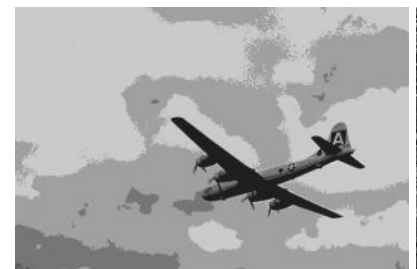

$6 / 0.947$

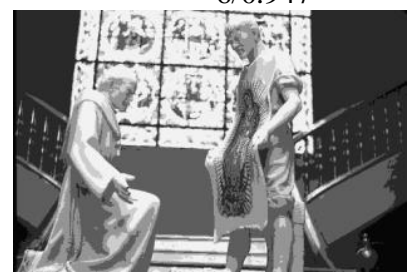

$6 / 0.986$

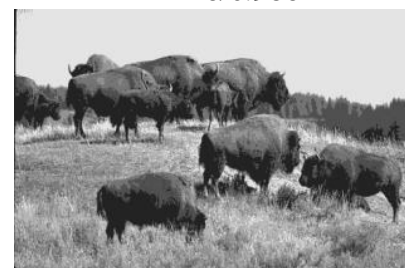

$6 / 0.986$

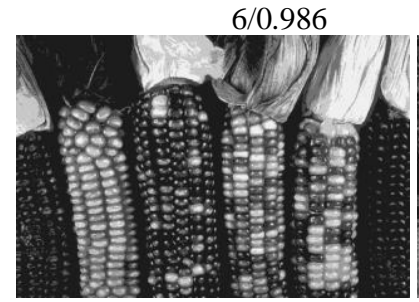

$6 / 0.986$

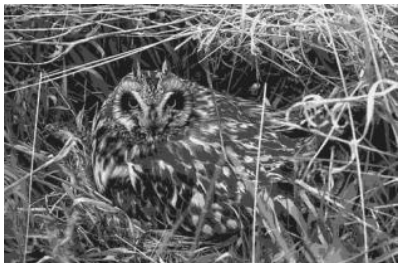

$6 / 0.979$

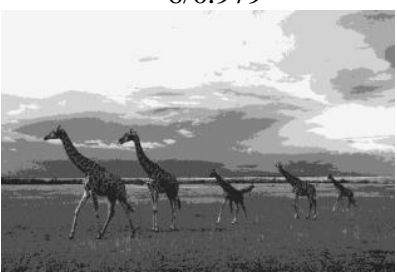

$6 / 0.988$

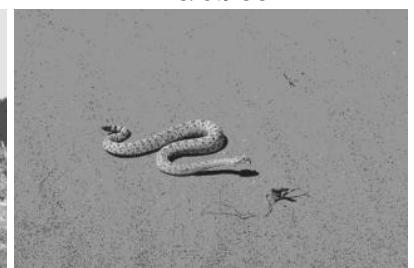

$6 / 0.807$

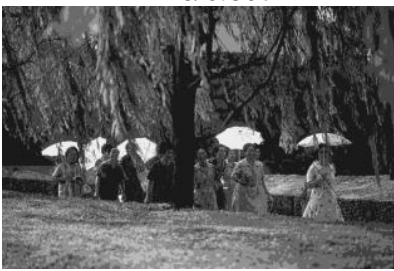

$6 / 0.974$

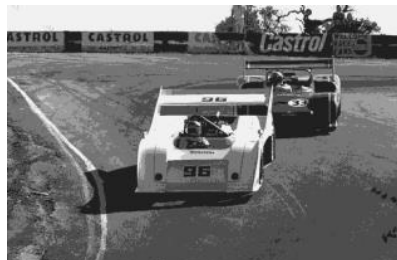

6/0.982

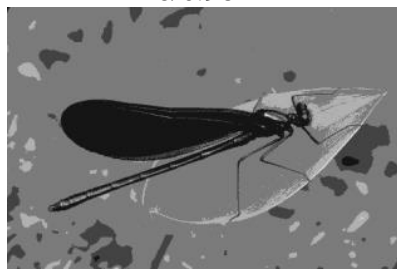

$5 / 0.925$

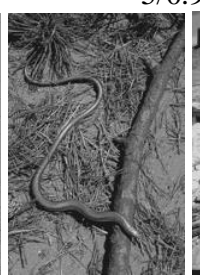

6/0.966

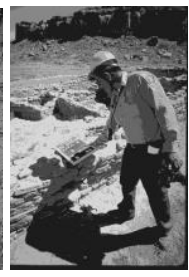

6/0.987

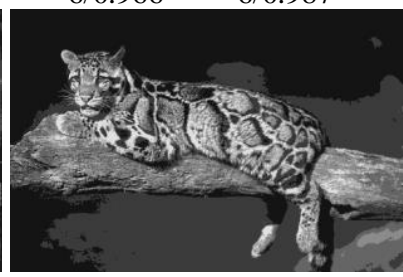

$6 / 0.978$

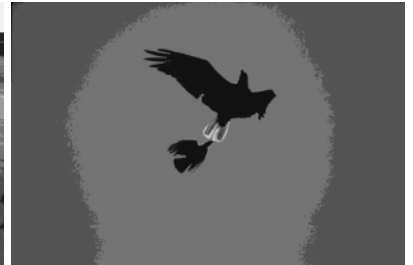

7/0.930

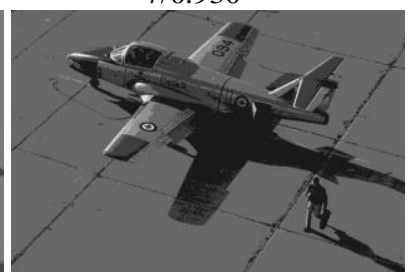

$6 / 0.962$

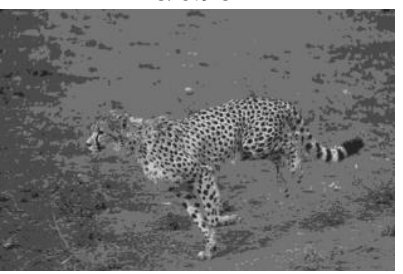

7/0.903

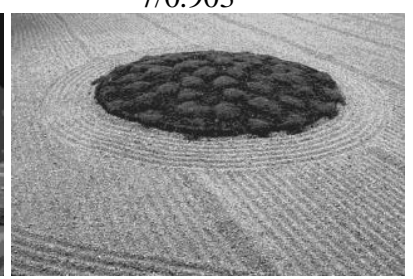

$6 / 0.969$

Fig. 15: Segmentation results applying third derivatives of a 25 order polynomial in tanh function for some image from BSD having \#regions/SSIM shown below each image

Table 1: Procedure of the Proposed Algorithm

1. Read Image.

2. Normalize Image to $[0,1]$.

3. Find mean and std.

4. Find normalized gray values using Equation (1).

5. Find 1D histogram, pick non-zero bins only. Normalize the histogram to have a sum of one. call it $h_{\text {org. }}$

6. Construct a polynomial of order $N$ using the tanh function as basis, see Equation (2).

7. The derivative of the polynomial in STEP 6 is fitted to $h_{\text {org. }}$.

8. Solve the $N \times N$ linear system resulting from Equation (5).

9. Construct the fitted histogram $h_{\text {fit }}$ using the coefficients found in STEP 8.

10. The minima of $h_{\text {fit }}$ are the thresholds.

11. Segment the image according to the thresholds found in STEP 10.

Another comparison is performed with $\mathrm{Hu}$ et al. (2019). Artificial Bee Colony (ABC) is used to find multi-level thresholds that minimize the normalized graph cuts. To save space, images will not be shown. Table 2 lists the values of RSME and SSIM for some images resulting from the proposed scheme and that of Hu et al. (2019).

As expected, small values of $\mathrm{N}$ will not be able to accurately follow the histogram curve. However, the proposed scheme was able to produce better performance (in terms of SSIM) than the scheme in Hu et al. (2019) (a meta heuristic scheme) as clearly indicated in Table 2. Even though RMSE has a weak relation with the human visual system, comparison shows that the proposed scheme has lower RMSE for segmentations with a larger number of regions.

In fact, the proposed scheme has the advantage of suggesting the best number of regions if large values of $\mathrm{N}$ were used $(\geq 20)$ together with the third or higher odd derivatives of the fitting polynomial. The reason is that 
higher derivatives can detect small regions buried under the shoulders and long tails of a larger region.

Figure 15 shows some segmented images from BSD using an order of 25 and the third derivative.
Figure 16 shows some images where color is not required to distinguish regions in the image. Segmentation results are shown for an order of 25 and the third derivative.

Table 2: RMSE/SSIM for some standard images and from BSD obtained from the proposed scheme and that of Hu et al. (2019)

\begin{tabular}{|c|c|c|c|c|}
\hline Image & \#regions & Hu et al. (2019) & Proposed & $\mathrm{N} /$ derivative \\
\hline \multirow[t]{4}{*}{ Pepper } & 2 & $18.14 / 0.63$ & $30.00 / 0.873$ & $12 / 1$ \\
\hline & 3 & $17.41 / 0.78$ & $22.93 / 0.926$ & $14 / 1$ \\
\hline & 4 & $16.51 / 0.85$ & $17.10 / 0.960$ & $22 / 1$ \\
\hline & 5 & $16.11 / 0.94$ & $13.80 / 0.972$ & $25 / 1$ \\
\hline \multirow[t]{4}{*}{ Camera } & 2 & $18.06 / 0.71$ & $28.46 / 0.898$ & $5 / 1$ \\
\hline & 3 & $16.61 / 0.81$ & $22.40 / 0.949$ & $12 / 1$ \\
\hline & 4 & $16.04 / 0.94$ & $20.59 / 0.953$ & $8 / 3$ \\
\hline & 5 & $15.63 / 0.97$ & $13.59 / 0.978$ & $12 / 3$ \\
\hline \multirow[t]{4}{*}{24077} & 2 & $17.21 / 0.79$ & $37.92 / 0.863$ & $4 / 1$ \\
\hline & 3 & $16.92 / 0.84$ & $27.22 / 0.937$ & $6 / 1$ \\
\hline & 4 & $16.82 / 0.88$ & 19.86/0.967 & $10 / 1$ \\
\hline & 5 & $16.56 / 0.94$ & $15.38 / 0.981$ & $20 / 1$ \\
\hline \multirow[t]{4}{*}{89072} & 2 & $18.10 / 0.62$ & $32.05 / 0.868$ & $4 / 1$ \\
\hline & 3 & $18.02 / 0.76$ & $21.17 / 0.946$ & $7 / 1$ \\
\hline & 4 & $17.34 / 0.82$ & $15.68 / 0.971$ & $12 / 1$ \\
\hline & 5 & $16.96 / 0.88$ & $12.38 / 0.982$ & $20 / 1$ \\
\hline \multirow[t]{4}{*}{42049} & 2 & $18.03 / 0.76$ & $25.96 / 0.905$ & $4 / 1$ \\
\hline & 3 & $17.72 / 0.83$ & $17.23 / 0.959$ & $7 / 1$ \\
\hline & 4 & $16.72 / 0.92$ & $12.38 / 0.978$ & $12 / 1$ \\
\hline & 5 & $15.90 / 0.97$ & $10.85 / 0.983$ & $20 / 1$ \\
\hline \multirow[t]{4}{*}{86016} & 2 & $18.00 / 0.54$ & $30.32 / 0.832$ & $4 / 1$ \\
\hline & 3 & $17.45 / 0.66$ & $22.19 / 0.914$ & $6 / 1$ \\
\hline & 4 & $17.21 / 0.73$ & $16.56 / 0.948$ & $9 / 3$ \\
\hline & 5 & $15.69 / 0.92$ & $13.41 / 0.965$ & $11 / 3$ \\
\hline \multirow[t]{4}{*}{54082} & 2 & $17.92 / 0.32$ & $30.76 / 0.756$ & $4 / 1$ \\
\hline & 3 & $17.11 / 0.38$ & $18.41 / 0.886$ & $6 / 1$ \\
\hline & 4 & $15.33 / 0.60$ & $19.12 / 0.886$ & $8 / 3$ \\
\hline & 5 & $14.76 / 0.92$ & $13.22 / 0.941$ & $12 / 3$ \\
\hline \multirow[t]{4}{*}{21077} & 2 & $17.06 / 0.48$ & $35.30 / 0.805$ & $4 / 1$ \\
\hline & 3 & $16.35 / 0.73$ & $24.27 / 0.910$ & $5 / 1$ \\
\hline & 4 & $16.12 / 0.89$ & $17.16 / 0.956$ & $10 / 1$ \\
\hline & 5 & $15.15 / 0.93$ & $13.73 / 0.972$ & $15 / 3$ \\
\hline \multirow[t]{4}{*}{160068} & 2 & $18.21 / 0.61$ & $31.95 / 0.836$ & $4 / 1$ \\
\hline & 3 & $17.89 / 0.67$ & $22.16 / 0.916$ & $5 / 1$ \\
\hline & 4 & $16.61 / 0.72$ & $20.24 / 0.928$ & $15 / 3$ \\
\hline & 5 & $15.59 / 0.77$ & $13.24 / 0.971$ & $20 / 3$ \\
\hline \multirow{4}{*}{37073} & 2 & $18.50 / 0.33$ & $33.82 / 0.813$ & $9 / 1$ \\
\hline & 3 & $18.09 / 0.66$ & $17.40 / 0.903$ & $10 / 1$ \\
\hline & 4 & $16.27 / 0.82$ & $11.43 / 0.928$ & $5 / 3$ \\
\hline & 5 & $15.41 / 0.89$ & $8.71 / 0.960$ & $20 / 3$ \\
\hline \multirow[t]{4}{*}{66053} & 2 & $18.47 / 0.47$ & $28.99 / 0.778$ & $4 / 1$ \\
\hline & 3 & $18.01 / 0.79$ & $20.32 / 0.911$ & $10 / 1$ \\
\hline & 4 & $16.89 / 0.84$ & $18.44 / 0.924$ & $20 / 1$ \\
\hline & 5 & $16.31 / 0.88$ & $15.50 / 0.945$ & $12 / 3$ \\
\hline \multirow[t]{4}{*}{304074} & 2 & $17.72 / 0.55$ & $33.54 / 0.814$ & $4 / 1$ \\
\hline & 3 & $17.28 / 0.69$ & $20.14 / 0.926$ & $5 / 1$ \\
\hline & 4 & $16.30 / 0.78$ & $15.97 / 0.954$ & $9 / 3$ \\
\hline & 5 & $16.01 / 0.86$ & $13.17 / 0.969$ & $15 / 3$ \\
\hline
\end{tabular}



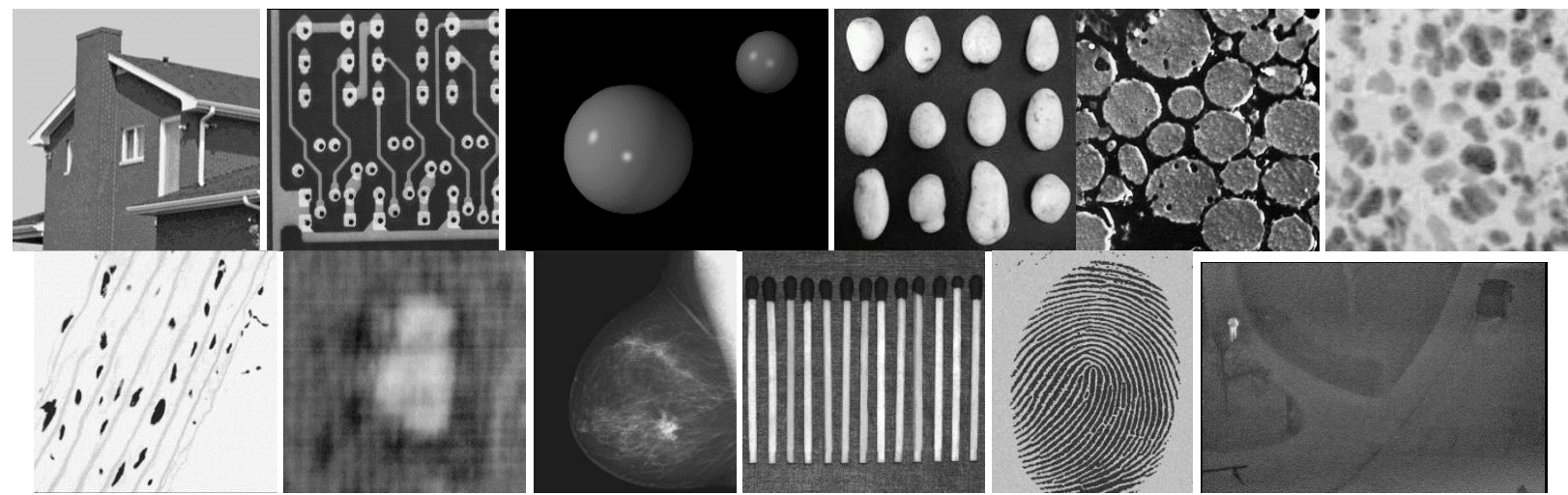

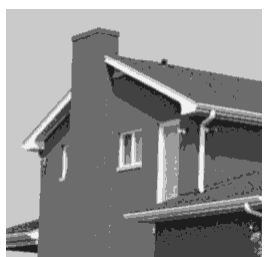

$6 / 0.991$

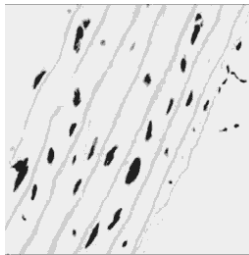

$6 / 0.960$

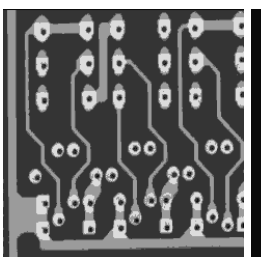

$5 / 0.984$

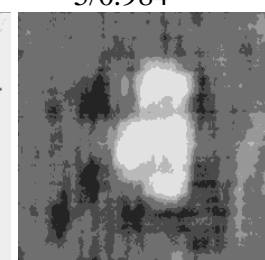

$6 / 0.968$

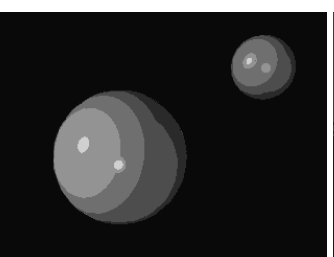

$6 / 0.954$

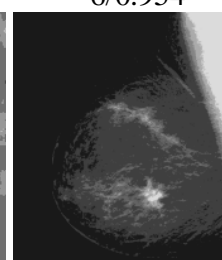

$6 / 0.967$

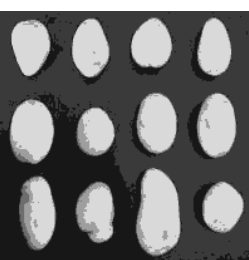

$5 / 0.989$

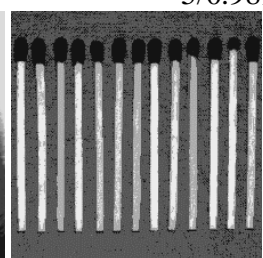

$4 / 0.948$

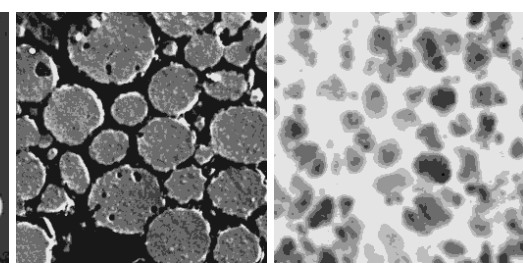

$6 / 0.979$

$6 / 0.975$

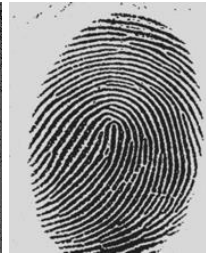

$4 / 0.990$

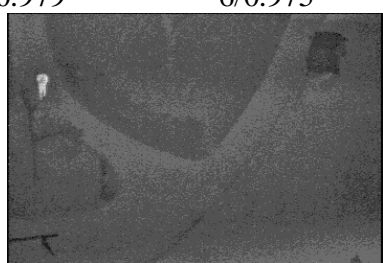

$8 / 0.911$

Fig. 16: Segmentation results applying third derivatives of a 25 order polynomial in tanh function for images where color is not required in regions' description. (left to right and top to buttom) having \#regions/SSIM shown below each segmented image. Original Images are shown in the top 2 rows

\section{Conclusion and Future Work}

A novel scheme for multi-level image thresholding has been proposed. The derivative of a polynomial in tanh function (similar argument to $\tan ^{-1}$ and erf) is used to fit the histogram. It has been found experimentally to be better than fitting the cumulative histogram to the original polynomial or the cumulative sum of its derivative.

A Lagrange optimization scheme may help in combining both, to be formulated in a future work. The Lagrange formulation can also help in regularizing the polynomial coefficients, as their high values indicate an ill-conditioned linear system.

The polynomial order can be carefully chosen to specify the number of the thresholds. However, this is highly image dependent.

Higher order derivatives were used to separate regions in the image with overlapping histograms (a shoulder for example).

Results are promising, however, more test images are needed to explore the limits of the proposed scheme. The domain of application as well as extensions to higher dimensional histograms are currently under investigation.

\section{Ethics}

This article is original and contains unpublished material. The corresponding author confirms that all of the other authors have read and approved the manuscript and no ethical issues involved.

\section{References}

Balabanian, F., E. da Silva and H. Pedrini, 2017. Image thresholding improved by global optimization methods. Applied Artificial Intell., 31: 197-208. DOI: $10.1080 / 08839514.2017 .1300050$

Hu, Y., J. Wang, X. Ai and X. Zhuang, 2019. An improved multithreshold segmentation algorithm based on graph cuts applicable for irregular image. Math. Problems Eng. DOI: $10.1155 / 2019 / 3514258$ 
Kapur, J., P. Sahoo and A. Wong, 1985. A new method for gray-level picture thresholding using the entropy of the histogram. Comput. Vis. Graph. Image Process., 29: 273-285. DOI: 10.1016/0734-189X(85)90125-2

Liu, D. and J. Yu, 2009. Otsu method and K-means. Proceedings of the 9th International Conference on Hybrid Intelligent Systems, Aug. 12-14, IEEE Xplore Press, Shenyang, China, pp: 344-349.

DOI: 10.1109/HIS.2009.74

Martin, D., C. Fowlkes, D. Tal and J. Malik, 2001. A database of human segmented natural images and its application to evaluating segmentation algorithms and measuring ecological statistics. Proceedings of the 8th IEEE International Conference on Computer Vision, Jul. 7-14, IEEE Xplore Press, Vancouver, BC, Canada, pp: 416-423.

DOI: $10.1109 /$ ICCV.2001.937655

Merzban, M. and M. Elbayoumi, 2019. Efficient solution of Otsu multilevel image thresholding: A comparative study. Expert Syst. Applic., 116: 299-309.

DOI: 10.1016/j.eswa.2018.09.008

Oliva, D., S. Hinojosa, M. Abd Elaziz and N. Sánchez, 2018. Context based image segmentation using antlion optimization and sine cosine algorithm. Multimed. Tools Applic., 77: 25761-25797.

DOI: $10.1007 / \mathrm{S} 11042-018-5815-\mathrm{X}$.
Olugbara, O., E. Adetiba and S. Oyewole, 2015. Pixel intensity clustering algorithm for multilevel image segmentation. Math. Problems Eng.

DOI: $10.1155 / 2015 / 649802$

Otsu, N., 1979. A threshold selection method from graylevel histograms. IEEE Trans. Syst. Man. Cybernet., 9: 62-66. DOI: 10.1109/TSMC.1979.4310076

Patra, S., R. Gautamb and A. Singla, 2014. A novel context sensitive multilevel thresholding for image segmentation. Applied Soft Comput., 23: 122-127. DOI: 10.1016/j.asoc.2014.06.016

Sezgin, M. and B. Sankur, 2004. Survey over image thresholding techniques and quantitative performance evaluation. J. Electronic Imag., 13: 146-165. DOI: 10.1117/1.1631315

Wang, Z., A. Bovik, H. Sheikh and E. Simoncelli, 2004. Image quality assessment: From error visibility to structural similarity. IEEE Trans. Image Process., 13: 1-14. DOI: 10.1109/TIP.2003.819861 